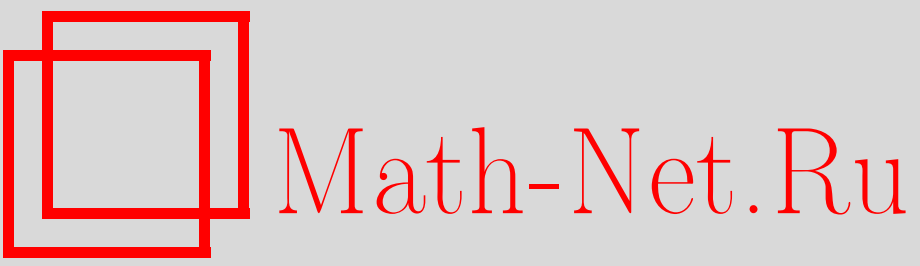

Ю. Н. Тюрин, Многомерный статистический анализ: геометрическая теория, Теория вероятн. и ее примен., 2010, том 55, выпуск 1, 36-58

DOI: https://doi.org/10.4213/tvp4175

Использование Общероссийского математического портала Math-Net.Ru подразумевает, что вы прочитали и согласны с пользовательским соглашением

http://www . mathnet.ru/rus/agreement

Параметры загрузки:

IP : 54.197 .130 .99

26 апреля 2023 г., 14:29:01 


\title{
МНОГОМЕРНЫЙ СТАТИСТИЧЕСКИЙ АНАЛИЗ: ГЕОМЕТРИЧЕСКАЯ ТЕОРИЯ ${ }^{1)}$
}

\begin{abstract}
В статье построена геометрическая теория многомерного статистического анализа, в основных чертах воспроизводящая классическую одномерную теорию. Как инструмент использована построенная здесь же расширенная (по сравнению с классической) форма линейной алгебры, в которой скалярными произведениями элементов служат квадратные матрицы. В терминах этой обобщенной линейной алгебры даны общие понятия линейных моделей и линейных гипотез; предложен матричный метод наименьших квадратов и указана его связь с традиционным; развит общий метод для проверки линейных статистических гипотез в геометрической бескоординатной форме.
\end{abstract}

Ключевые слова и фразы: многомерный статистический анализ, таблицы, модуль таблиц над кольцом матриц, матричнозначное скалярное произведение таблиц, многомерные линейные модели, многомерные линейные гипотезы, матричный метод наименьших квадратов, распределение Уишарта.

Введение. Линейный статистический анализ и метод наименьших квадратов как его часть получили свою современную завершенную форму на языке линейной алгебры, т.е. на языке геометрии. В этой статье мы намерены показать, что и многомерный линейный статистический анализ на геометрическом языке может быть изложен столь же красиво и ясно. Для этого стандартные средства линейной алгебры должны быть расширены. Изложению этого обобщения линейной алгебры отведен первый раздел статьи. Во втором разделе статьи излагается теория многомерного статистического анализа на языке, разработанном в первом. На наш взгляд, до сих пор многомерный статистический анализ, хоть и изложен в десятках учебников, не имел адекватных средств выражения.

Многомерное наблюдение - это наблюдаемые в одном случайном эксперименте несколько случайных величин. Многомерные наблюдения

\footnotetext{
* Московский государственный университет им. М. В. Ломоносова, механико-математический факультет, кафедра теории вероятностей, Ленинские горы, 119991 Москва, Россия; e-mail: ytjurin@gmail.ru

1) Работа выполнена при финансовой поддержке РФФИ (грант № 06-01-00454).
} 
мы далее записываем в виде столбцов. Многомерные наблюдения (как и одномерные) мы обычно снабжаем индексами. В простейшем случае индексами служат натуральные числа. (Это могут быть номера наблюдений в порядке их регистрации.) Для независимых одинаково распределенных наблюдений это подходящий способ организации данных. Если распределения наблюдений зависят от одного или нескольких факторов, индексами могут служить значения или комбинации значений этих факторов. Впрочем, обычно уровни факторов тоже снабжают номерами. В этом случае индекс - это набор чисел. Так, в двухфакторной схеме (классификация по двум признакам) индексами служат пары натуральных чисел.

Совокупность наблюдений, снабженных индексами, мы будем называть таблицей.

Для теоретического анализа наиболее удобна линейная нумерация данных. Далее мы будем придерживать этой системы. При рассмотрении примеров мы будем возвращаться, если потребуется, к естественной индексации данных.

В одномерном статистическом анализе нумерация данных предлагает их запись в виде строки. В многомерном случае совокупность нумерованных данных, т.е. таблиц, мы также будем воспринимать как строку, позиции в которой заняты столбцами. Во многих случаях (но не всегда!) такую таблицу можно воспринимать как матрицу.

Таблицы одной формы естественно образуют векторное пространство относительно операций сложения и умножения на числа. Для целей статистического анализа это векторное пространство наделяют скалярным произведением. В одномерном анализе если наблюдения независимы и имеют одинаковые дисперсии, то наиболее подходящим скалярным произведением является евклидово произведение. Более подробно: пусть наблюдения снабжены индексом $\alpha$; пусть таблицы $\mathbf{T}_{X}$ и $\mathbf{T}_{Y}$ состоят из одномерных элементов $X_{\alpha}, Y_{\alpha}$ соответственно. Тогда евклидово скалярное произведение таблиц $\mathbf{T}_{X}$ и $\mathbf{T}_{Y}$ есть

$$
\left\langle\mathbf{T}_{X}, \mathbf{T}_{Y}\right\rangle=\sum_{\alpha} X_{\alpha} Y_{\alpha}
$$

где индекс суммирования пробегает все значения.

Многомерные наблюдения мы записываем в виде столбцов. В многомерном случае элементы $X_{\alpha}, Y_{\alpha}$ являются столбцами. Для таблиц, элементами которых являются столбцы, примем такое определение скалярного произведения таблиц $\mathbf{T}_{X}$ и $\mathbf{T}_{Y}$ :

$$
\left\langle\mathbf{T}_{X}, \mathbf{T}_{Y}\right\rangle=\sum_{\alpha} X_{\alpha} Y_{\alpha}^{T}
$$

Скалярное произведение $(*)$ - это квадратная матрица. Следовательно, для таблиц, элементами которых являются столбцы, квадрат- 
ные матрицы соответствующей размерности должны играть роль скаляров. С помощью скалярного произведения $(*)$ и его применений в статье будет построена теория многомерного статистического анализа, в основных чертах параллельная сложившейся одномерной теории.

\section{1. Модуль таблиц над кольцом квадратных матриц.}

1.1. Пространство таблиц. Во введении мы условились ради упрощения и единообразия теоретического анализа придерживаться линейного порядка индексации (т.е. нумерации) наблюдений. Однако все вводимые нами понятия и связывающие их теоремы с очевидными изменениями остаются верными и для таблиц с иной индексацией.

Рассмотрим $p$-мерную таблицу с $n$ элементами

$$
\mathbf{T}:=\left\{X_{i} \mid i=1, \ldots, n\right\},
$$

где $X_{1}, \ldots, X_{n}$ суть $p$-мерные векторы-столбцы. Таблицы такой формы естественно образуют линейное пространство относительно операций сложения и умножения на числа.

\section{1. Сложение:}

$$
\left\{X_{i} \mid i=1, \ldots, n\right\}+\left\{Y_{i} \mid i=1, \ldots, n\right\}=\left\{X_{i}+Y_{i} \mid i=1, \ldots, n\right\} .
$$

2. Умножение на числа: пусть $\lambda-$ число; тогда

$$
\lambda\left\{X_{i} \mid i=1, \ldots, n\right\}=\left\{\lambda X_{i} \mid i=1, \ldots, n\right\} .
$$

Помимо умножения на числа, мы будем рассматривать поэлементное умножение таблиц на квадратные матрицы соответствующих размеров.

3. Умножение на матрицы слева: пусть $K-$ квадратная матрица размерности $p \times p$. Положим

$$
K\left\{X_{i} \mid i=1, \ldots, n\right\}=\left\{K X_{i} \mid i=1, \ldots, n\right\} .
$$

Отметим, что умножение таблицы на число можно рассматривать как частный случай умножения на квадратную матрицу. А именно: умножение на число $\lambda$ есть умножение на матрицу $\lambda I$, где $I-$ единичная матрица размера $p \times p$.

4. Умножение на матрицы справа: пусть $Q=\| q_{i j} \mid i=$ $1, \ldots, n, j=1, \ldots, n \|-$ квадратная $(n \times n)$-матрица. Определим умножение таблицы $\mathbf{T}(1.1)$ на матрицу $Q$ справа, положив

$$
\left\{X_{i} \mid i=1, \ldots, n\right\} Q=\left\{\sum_{j=1}^{n} X_{j} q_{i j} \mid i=1, \ldots, n\right\} .
$$


Видно, что произведение $\mathbf{T} Q$ определено обычным способом по правилу «строка на столбец» с той лишь разницей, что элементами строки $\mathbf{T}$ (таблицы $\mathbf{T}$ ) служат не числа, но столбцы $X_{1}, \ldots, X_{n}$.

5. Определим в пространстве таблиц внутреннее произведение. За его свойства и последующее употребление будем называть его скалярным произведением (точнее, обобщенным скалярным произведением). Более подробно: пусть

$$
\mathbf{T}=\left\{X_{i} \mid i=1, \ldots, n\right\}, \quad \mathbf{R}=\left\{Y_{i} \mid i=1, \ldots, n\right\} .
$$

Скалярным (обобщенным скалярным) произведением таблии $\mathbf{T}$ и $\mathbf{R}$ назовем

$$
\langle\mathbf{T}, \mathbf{R}\rangle=\sum_{i=1}^{n} X_{i} Y_{i}^{T}
$$

Результатом произведения является квадратная $(p \times p)$-матрица. Скалярное произведение некоммутативно:

$$
\langle\mathbf{R}, \mathbf{T}\rangle=\langle\mathbf{T}, \mathbf{R}\rangle^{T} .
$$

6. Скалярный квадрат таблицы Т есть симметричная неотрицательно определенная $(p \times p)$-матрица

$$
\langle\mathbf{T}, \mathbf{T}\rangle=\sum_{i=1}^{n} X_{i} X_{i}^{T}
$$

Для обозначения скалярного квадрата будем употреблять привычный символ абсолютного значения $\langle\mathbf{T}, \mathbf{T}\rangle=|\mathbf{T}|^{2}$. В нашем случае $|\mathbf{T}|$ - это так называемый матричный модуль.

7. Свойства скалярного произведения в пространствах таблиц напоминают свойства традиционного скалярного произведения в евклидовых векторных пространствах: если $\mathbf{T}_{1}, \mathbf{T}_{2}, \mathbf{T}_{3}$ - это таблицы общей формы, то

$$
\begin{aligned}
& \left\langle\mathbf{T}_{1}+\mathbf{T}_{2}, \mathbf{T}_{3}\right\rangle=\left\langle\mathbf{T}_{1}, \mathbf{T}_{3}\right\rangle+\left\langle\mathbf{T}_{2}, \mathbf{T}_{3}\right\rangle ; \\
& \left\langle K \mathbf{T}_{1}, \mathbf{T}_{2}\right\rangle=K\left\langle\mathbf{T}_{1}, \mathbf{T}_{2}\right\rangle, \text { если } K-\text { квадратная }(p \times p) \text {-матрица; } \\
& \left\langle\mathbf{T}_{1}, \mathbf{T}_{1}\right\rangle \succcurlyeq 0 \text { в смысле сравнения квадратных симметричных матриц; } \\
& \left\langle\mathbf{T}_{1}, \mathbf{T}_{1}\right\rangle=0 \text { тогда и только тогда, когда } \mathbf{T}_{1}=0 .
\end{aligned}
$$

8. Мы скажем, что таблица $\mathbf{T}$ ортогональна таблице $\mathbf{R}$, если

$$
\langle\mathbf{T}, \mathbf{R}\rangle=0 .
$$

Заметим, что если $\langle\mathbf{T}, \mathbf{R}\rangle=0$, то и $\langle\mathbf{R}, \mathbf{T}\rangle=0$. Поэтому свойство ортогональности таблиц является взаимным. Ортогональность таблиц $\mathbf{T}$ и $\mathbf{R}$ будем обозначать $\mathbf{T} \perp \mathbf{R}$. 
9. Отметим форму теоремы Пифагора: если таблицы $\mathbf{T}$ и $\mathbf{R}$ ортогональны, то

$$
\langle\mathbf{T}+\mathbf{R}, \mathbf{T}+\mathbf{R}\rangle=\langle\mathbf{T}, \mathbf{T}\rangle+\langle\mathbf{R}, \mathbf{R}\rangle
$$

Еще раз отметим, что результатом скалярного произведения двух таблиц является $(p \times p)$-матрица, поэтому в пространствах таблиц квадратные матрицы соответствующих размеров должны играть роль скаляров. В частности, умножение на $(p \times p)$-матрицу слева мы будем рассматривать как умножение на скаляр и таблицу $K \mathbf{T}$ будем считать пропорциональной таблице $\mathbf{T}$.

Обозначения. Матрицы с числовыми элементами, у которых $p$ строк и $n$ столбцов, будем называть $(p \times n)$-матрицами. Совокупность $(p \times n)$-матриц обозначаем $\mathbb{R}_{n}^{p}$. Матрицы размера $p \times 1$ будем называть $p$-столбцами или просто столбцами. Совокупность $p$-столбцов обозначим $\mathbb{R}_{1}^{p}$. Матрицы $1 \times n$ называем $n$-строками или просто строками. Совокупность $n$-строк обозначаем $\mathbb{R}_{n}^{1}$.

10. Вместе с таблицами вида (1.1) будем рассматривать взаимно однозначно соответствующие им матрицы

$$
x=\left\|X_{1}, \ldots, X_{n}\right\|
$$

Матрица (1.7) - это матрица с $p$ строками и $n$ столбцами.

Можно сказать, что пространство таблиц вида (1.1) это тензорное произведение $\mathbb{R}_{1}^{p} \otimes \mathbb{R}_{1}^{n}$. С алгебраической точки зрения пространство таблиц с введенными выше операциями - это модуль над кольцом квадратных матриц соответствующего размера (в данном случае над кольцом $(p \times p)$-матриц $)$. В этом модуле определена бинарная операция со значениями в упомянутом кольце. Эту операцию из-за ее свойств мы назвали скалярным произведением, ибо элементы кольца для модуля таблиц играют роль скаляров.

Многие операции с таблицами можно проводить в их матричных формах. В частности, сложению таблиц соответствует сложение их матриц; левому умножению на квадратную $(p \times p)$-матрицу $K$ соответствует матричное произведение $K X$; умножению (справа) на матрицу $Q$ соответствует матричное произведение $X Q$; скалярное произведение таблиц

$$
\mathbf{T}_{X}=\left\{X_{i} \mid i=1, \ldots, n\right\} \quad \text { и } \quad \mathbf{T}_{Y}=\left\{Y_{i} \mid i=1, \ldots, n\right\}
$$

равно произведению соответствующих им матриц $\mathcal{X}$ и $y$ :

$$
\left\langle\mathbf{T}_{X}, \mathbf{T}_{Y}\right\rangle=x y^{T} .
$$

Все эти соотношения проверяются прямыми выкладками. 
1.2. Линейные преобразования. Многие понятия классической линейной алгебры почти автоматически переносятся на пространства таблиц - с естественным расширением запаса скаляров до кольца квадратных матриц.

В частности, преобразование $f(\cdot)$ пространства таблиц (1.1) в себя естественно назвать линейныл, если для любых таблиц $\mathbf{T}_{1}$ и $\mathbf{T}_{2}$ и для любых $(p \times p)$-матриц $K_{1}$ и $K_{2}$

$$
f\left(K_{1} \mathbf{T}_{1}+K_{2} \mathbf{T}_{2}\right)=K_{1} f\left(\mathbf{T}_{1}\right)+K_{2} f\left(\mathbf{T}_{2}\right) .
$$

Линейные преобразования в пространствах таблиц осуществляются умножением справа на квадратные матрицы. Пусть $Q=\left\|q_{i j}\right\|-$ произвольная $(n \times n)$-матрица, $\mathbf{T}-$ произвольная таблица (1.1). То, что преобразования

$$
f(\mathbf{T})=\mathbf{T} Q
$$

являются линейными в смысле (1.8), непосредственно следует из определения (1.3). То, что других линейных преобразований нет, следует из того, что их нет даже в случае $p=1$. (Как известно, все линейные преобразования в векторных пространствах строк осуществляются умножением справа на квадратные $(n \times n)$-матрицы.)

Отметим, что матричная форма (1.7) представления таблиц подходит и для описания линейных преобразований: матрица $X Q$ (произведение матриц $\mathcal{X}$ и $Q$ ) совпадает с матричной формой таблицы (1.3)

$$
\mathbf{T} Q=\left\{X_{i} \mid i=1, \ldots, n\right\} Q=\left\{\sum_{j=1}^{n} X_{j} q_{i j} \mid i=1, \ldots, n\right\} .
$$

Линейное преобразование (пространства таблиц в себя) мы будем называть ортогональным, если это преобразование сохраняет скалярное произведение, а именно если для таблиц $\mathbf{T}$ и $\mathbf{R}$

$$
\langle\mathbf{T} Q, \mathbf{R} Q\rangle=\langle\mathbf{T}, \mathbf{R}\rangle .
$$

Легко видеть, что ортогональное преобразование осуществляется умножением справа на ортогональную матрицу. Действительно,

$$
\begin{aligned}
\langle\mathbf{T} Q, \mathbf{R} Q\rangle & =\sum_{i=1}^{n}\left(\sum_{j=1}^{n} X_{j} q_{i j}\right)\left(\sum_{l=1}^{n} Y_{l} q_{i l}\right)^{T} \\
& =\sum_{j=1}^{n} \sum_{l=1}^{n} X_{j} Y_{l}^{T}\left(\sum_{i=1}^{n} q_{i j} q_{i l}\right)=\sum_{j=1}^{n} X_{j} Y_{j}^{T},
\end{aligned}
$$

поскольку матрица $Q$ ортогональна и потому $\sum_{i=1}^{n} q_{i j} q_{i l}=\delta_{j l}$ (символ Кронекера). 
1.3. Порождающие базисы и координаты. Особую роль в пространстве таблиц (1.1), когда они представлены в виде $(p \times n)$ матриц (1.7), играют $(p \times n)$-матрицы вида $\alpha x$, где $\alpha$ - это $p$-столбец, $x$ - это $n$-строка, символ $\alpha x$ обозначает произведение матриц $\alpha \in \mathbb{R}_{1}^{p}$, $x \in \mathbb{R}_{n}^{1}$.

Пусть $n$-строки $e_{1}, \ldots, e_{n} \in \mathbb{R}_{n}^{1}$ образуют базис пространства $\mathbb{R}_{n}^{1}$. Пусть $\alpha_{1}, \ldots, \alpha_{n} \in \mathbb{R}_{1}^{p}$ - произвольные $p$-столбцы. Рассмотрим $(p \times n)$ матрицы $\alpha_{1} e_{1}, \ldots, \alpha_{n} e_{n}$.

Теорема 1. Всякую $(p \times n)$-матрииу $X(1.7)$ можно представить в виде

$$
X=\sum_{i=1}^{n} \alpha_{i} e_{i}
$$

при некотором выборе $\alpha_{1}, \ldots, \alpha_{n} \in \mathbb{R}_{1}^{p}$, и притом единственным образом.

Д о к а з а т е ль с т в о. Определим матрицу $E$ размера $n \times n$, образованную $n$-строками $e_{1}, \ldots, e_{n}$. Введем также переменную матрицу $A$ размера $p \times n$, образованную $p$-столбцами $\alpha_{1}, \ldots, \alpha_{n}$. С помощью матриц $A$ и $E$ сумма (1.9) может быть записана как

$$
\sum_{i=1}^{n} \alpha_{i} e_{i}=A E
$$

Вот вычисления, подтверждающие это утверждение. Пусть $\alpha_{i}=$ $\left(\alpha_{1 i}, \ldots, \alpha_{p i}\right)^{T}, e_{i}=\left(e_{i 1}, \ldots, e_{i n}\right)$. Тогда

$$
\sum_{i=1}^{n} \alpha_{i} e_{i}=\sum_{i=1}^{n}\left(\alpha_{1 i}, \ldots, \alpha_{p i}\right)^{T}\left(e_{i 1}, \ldots, e_{i n}\right)
$$

Элементы каждого из $n$ произведений $\alpha_{i} e_{i}$, стоящие на месте $(k, l)$, суть $\alpha_{k i} e_{i l}$. Их общая сумма, т.е. элемент матрицы $\sum_{i=1}^{n} \alpha_{i} e_{i}$, равна $\sum_{i=1}^{n} \alpha_{k i} e_{i l}$.

Элемент матрицы $A E$, который стоит на месте $(k, l)$ (вычисляемый по правилу «строка на столбец»), равен $\sum_{i=1}^{n} \alpha_{k i} e_{i l}$. Результаты вычислений совпали.

Теорема будет доказана, если мы покажем, что уравнение

$$
X=A E
$$

имеет решение относительно $(p \times n)$-матрицы $A$, и притом единственное. Поскольку $(n \times n)$-матрица $E$ невырождена, это решение очевидно:

$$
A=x E^{-1} .
$$

Теорема 1 доказана. 
Теорема позволяет говорить, что базис в $\mathbb{R}_{n}^{1}$ порождает (указанным выше способом) все пространство $\mathbb{R}_{n}^{p}$. Поэтому базисы в $\mathbb{R}_{n}^{1}$ мы будем применительно к $\mathbb{R}_{n}^{p}$ называть порождаюшими базисами. При этом $p$-столбцы $\alpha_{1}, \ldots, \alpha_{n}$ из разложения $(1.9)(p \times n)$-матрицы $X$ по порождающему базису $e_{1}, \ldots, e_{n}$ можно рассматривать как координаты $x$ в этом базисе. Для канонического базиса пространства $\mathbb{R}_{n}^{1}$ (когда $e_{i}-$ это $n$-строка, в которой единица стоит на месте $i$, а прочие элементы равны нулю) координаты $X$ относительно этого базиса совпадают с $p$ столбцами $X_{1}, \ldots, X_{n} \in \mathbb{R}_{1}^{p}$, образующими матрицу $\mathcal{X}$.

Координаты таблицы в разных базисах. Координаты $(p \times n)$ матрицы $\mathcal{X}$ относительно разных порождающих базисов связаны линейным преобразованием. Пусть, например, $n$-строки $f_{1}, \ldots, f_{n}$ образуют базис $\mathbb{R}_{n}^{1}$. Пусть $F-(n \times n)$-матрица, составленная из этих $n$-строк. Согласно теореме 1 , существует и притом единственная совокупность $p$ столбцов $\beta_{1}, \ldots, \beta_{n}$ - координат $X$ относительно порождающего базиса $f_{1}, \ldots, f_{n}$. Матрицы $B=\left\|\beta_{1}, \ldots, \beta_{n}\right\|$ и $F$ связаны с $(p \times n)$-матрицей $X$ соотношением

$$
X=B F .
$$

Вместе с (1.10) это дает

$$
B F=A E .
$$

Отсюда

$$
B=A E F^{-1}, \quad A=B F E^{-1} .
$$

Следствие 1. Если порождающие базисы $e_{1}, \ldots, e_{n} u f_{1}, \ldots, f_{n}-$ ортогональнье, то переход от координат относительно одного базиса $\kappa$ координатам относительно другого базиса осуществляется умножением на ортогональную матричу.

Скалярные произведения в координатной форме. Рассмотрим в $\mathbb{R}_{n}^{p}$ произвольный ортогональный базис $e_{1}, \ldots, e_{n}$. Для произвольных $(p \times n)$-матриц $X$ и $y$ рассмотрим разложения (1.9) по этому базису:

$$
x=\sum_{i=1}^{n} \alpha_{i} e_{i}, \quad y=\sum_{i=1}^{n} \gamma_{i} e_{i} .
$$

Легко видеть, что

$$
\left\langle\mathbf{T}_{X}, \mathbf{T}_{Y}\right\rangle=x y^{T}=\sum_{i=1}^{n} \alpha_{i} \gamma_{i}^{T} .
$$

Равенство (1.12) означает, что в ортогональном базисе скалярное произведение равно попарному произведению координат. Действительно,

$$
x y^{T}=\left\langle\sum_{i=1}^{n} \alpha_{i} e_{i}, \sum_{j=1}^{n} \gamma_{j} e_{j}\right\rangle=\sum_{i=1}^{n} \sum_{j=1}^{n} \alpha_{i} e_{i} e_{j}^{T} \gamma_{j}^{T}=\sum_{i=1}^{n} \alpha_{i} \gamma_{i}^{T},
$$


так как для ортогонального базиса $e_{i} e_{j}^{T}=\delta_{i j}$. Следовательно, скалярный квадрат $\mathbf{T}_{X}$ равен

$$
\left|\mathbf{T}_{X}\right|^{2}=x X^{T}=\sum_{i=1}^{n} \alpha_{i} \alpha_{i}^{T}
$$

Получили, что квадрат длины (матричный модуль) произвольной таблицы равен сумме квадратов ее координат - совсем как квадрат длины вектора.

1.4. Подмодули. Подмодулем в пространстве таблиц (1.1) или соответствующих им матриц (1.7) назовем такое множество, которое замкнуто относительно линейных операций: сложения и умножения на скаляры (т.е. умножения слева на $(p \times p)$-матрицы). Для определенности далее будем говорить о таблицах в их матричных формах.

О п р е д е л е н и е 1. Множество $\mathscr{L} \subset \mathbb{R}_{n}^{p}$ назовем подмодулем пространства $\mathbb{R}_{n}^{p}$, если для любых $x_{1}, x_{2} \in \mathscr{L}$

$$
K_{1} x_{1}+K_{2} x_{2} \in \mathscr{L}
$$

при произвольных $(p \times p)$-матрицах $K_{1}, K_{2}$.

Теорема 2. Всякий подмодуль $\mathscr{L} \subset \mathbb{R}_{n}^{p}$ порождается некоторой линейно независимой системой $n$-строк. Число элементов в этой системе единственным образом определяется $\mathscr{L}$. Это число может быть названо размерностью подмодуля $\mathscr{L}$.

Д о к а з а т е л ь с т в о. Пусть $\mathcal{X} \in \mathscr{L}$. Множество $(p \times n)$-матриц вида $K \mathcal{X}$, где $K-$ произвольная $(p \times p)$-матрица, образует подмодуль. Обозначим его $\mathscr{L}(X)$. Пусть $x_{1}, \ldots, x_{p}$ - это $n$-строки $(p \times n)$-матрицы $x$. Выберем среди этих $n$-строк максимальную линейно независимую подсистему, скажем, $y_{1}, \ldots, y_{k}$. Очевидно, что

$$
\mathscr{L}(X)=\left\{y \mid y=\sum_{i=1}^{k} \beta_{i} y_{i}, \beta_{1}, \ldots, \beta_{k} \in \mathbb{R}_{1}^{p}\right\} .
$$

Если $\mathscr{L}(x)=\mathscr{L}$, то $y_{1}, \ldots, y_{k}$ составляют для $\mathscr{L} \subset \mathbb{R}_{n}^{p}$ порождающий базис. Если $\mathscr{L}(X) \neq \mathscr{L}$, то находим в $\mathscr{L}$ элемент, скажем, $\mathcal{Z}$, не принадлежащий $\mathscr{L}(X)$. Пополняем систему $y_{1}, \ldots, y_{k} n$-строками $z_{1}, \ldots, z_{p}(p \times n)$ матрицы $z$, находим в этой совокупности $n$-строк максимальную линейно независимую подсистему и т.д. На некотором шаге процесс остановится. Теорема 2 доказана.

Следствие 2. Всякий подмодуль $\mathscr{L} \subset \mathbb{R}_{n}^{p}$ может быть представлен как прямая сумма некоторых одномерных подмодулей $\mathscr{L}_{i} \subset \mathbb{R}_{n}^{p}$ :

$$
\mathscr{L}=\mathscr{L}_{1} \oplus \cdots \oplus \mathscr{L}_{l},
$$

гдe

$$
\mathscr{L}_{i}=\left\{X \mid X=\alpha y_{i}, \alpha \in \mathbb{R}_{1}^{p}\right\}
$$


для некоторого $y_{i} \in \mathbb{R}_{n}^{1}$. Число $l$ - одно и то же в любом представлении (1.13). Это число можно называть размерностью подмодуля $\mathscr{L}$ : $l=\operatorname{dim} \mathscr{L}$.

3 а м е ч а н и е. Линейно независимую систему $n$-строк, которая порождает $\mathscr{L}$, можно выбрать ортогональной. Для доказательства достаточно заметить, что порождающую систему можно превратить в ортогональную процессом ортогонализации.

Теорема 2 устанавливает взаимно однозначное соответствие между подмодулями векторного пространства $\mathbb{R}_{n}^{1}$ и пространства $(p \times n)$-матриц $\mathbb{R}_{n}^{p}$. Выделим этот факт как элемент теории в виде следующего утверждения.

Следствие 3. Каждому линейному пространству L в пространстве $n$-строк $\mathbb{R}_{n}^{1}$ соответствует некоторый подмодуль $\mathscr{L}$ в пространстве $(p \times n)$-матрич $\mathbb{R}_{n}^{p}$, и обратно. При этом размерности линейньи подпространств $L$ и подмодуля $\mathscr{L}$ совпадают.

Таким образом, пространство $\mathbb{R}_{n}^{p}$ (и соответствующее пространство таблиц) и пространство $\mathbb{R}_{n}^{1}$ располагают одинаковым «запасом» подмодулей и линейных подпространств. Это замечание имеет важные последствия для многомерного статистического анализа.

О п р е д е л е н и е 2 . Ортогональным дополнением к подмодулю $\mathscr{L}$ назовем множество

$$
\mathscr{L}^{\perp}=\left\{x \mid x \in \mathbb{R}_{n}^{p},\langle x, y\rangle=0 \forall y \in \mathscr{L}\right\}
$$

Легко видеть, что $\mathscr{L}^{\perp}$ является подмодулем и что

$$
\mathscr{L} \oplus \mathscr{L}^{\perp}=\mathbb{R}_{n}^{p}, \quad \operatorname{dim} \mathscr{L}^{\perp}=n-\operatorname{dim} \mathscr{L} .
$$

1.5. Проекции на подмодули. Рассмотрим пространство таблиц (1.1) с введенным в нем скалярным произведением (1.4). Пусть $\mathscr{L}$ - некоторый подмодуль. Назовем проекиией таблицы $\mathbf{T}$ на подмодуль $\mathscr{L}$ ту точку из $\mathscr{L}$, которая является ближайшей к $\mathbf{T}$ в смысле сравнения скалярных квадратов (1.5).

Скажем подробнее. Пусть таблица $\mathbf{R}$ пробегает множество $\mathscr{L}$. Мы называем точку $\mathbf{R}^{0} \in \mathscr{L}$ ближайшей $\kappa \mathbf{T}$, если для всякого $\mathbf{R} \in \mathscr{L}$

$$
\left\langle\mathbf{T}-\mathbf{R}^{0}, \mathbf{T}-\mathbf{R}^{0}\right\rangle \preccurlyeq\langle\mathbf{T}-\mathbf{R}, \mathbf{T}-\mathbf{R}\rangle .
$$

Обратим внимание, что $\langle\mathbf{T}-\mathbf{R}, \mathbf{T}-\mathbf{R}\rangle$ - это функция $\mathbf{R}$ со значениями в множестве матриц. Существование в множестве ее значений (при $\mathbf{R} \in \mathscr{L})$ минимального элемента не очевидно и не обеспечивается автоматически. Поэтому само существование $\operatorname{proj}_{\mathscr{L}} \mathbf{T}$ нуждается в доказательстве. Мы докажем следующую теорему. 
Теорема 3. Проекиия $\mathbf{T}$ на $\mathscr{L}$ существует, единственна и обладает привычныцми (по евклидовым пространствам) свойствами:

1) для любой табличь $\mathbf{R} \in \mathscr{L}$ справедливо

$$
|\mathbf{T}-\mathbf{R}|^{2} \succcurlyeq|\mathbf{T}-\underset{\mathscr{L}}{\operatorname{proj}} \mathbf{T}|^{2},
$$

причем равенство достигается только при $\mathbf{R}=\operatorname{proj}_{\mathscr{L}} \mathbf{T}$;

2) $\left(\mathbf{T}-\operatorname{proj}_{\mathscr{L}} \mathbf{T}\right) \perp \mathscr{L}$,

3) $\operatorname{proj}_{\mathscr{L}}\left(K_{1} \mathbf{T}_{1}+K_{2} \mathbf{T}_{2}\right)=K_{1} \operatorname{proj}_{\mathscr{L}} \mathbf{T}_{1}+K_{2} \operatorname{proj}_{\mathscr{L}} \mathbf{T}_{2}$.

Д ок аз а т ель с т в о этой теоремы дадим для матричного представления таблиц (1.7). Пусть $X \in \mathbb{R}_{n}^{p}$ - произвольная $(p \times n)$ матрица. (За подмодулем в пространстве $\mathbb{R}_{n}^{p}$, представляющим подмодуль таблиц, сохраним обозначение $\mathscr{L}$.) Как было показано, каждому подмодулю $\mathscr{L} \subset \mathbb{R}_{n}^{p}$ взаимно однозначно соответствует некоторое линейное подпространство $L$ в пространстве $n$-строк, $L \subset \mathbb{R}_{n}^{1}$. Пусть $\Pi-$ матрица оператора проектирования на $L$ в пространстве $\mathbb{R}_{n}^{1}$, т.е. для любого $x \in \mathbb{R}_{n}^{1}$

$$
\underset{\mathscr{L}}{\operatorname{proj}} x=x \Pi .
$$

Доказательство теоремы 3 вытекает из следующей леммы 1 и теоремы 4 .

Лемма 1. Пусть $\mathscr{L} \subset \mathbb{R}_{n}^{p}-$ подмодуль в пространстве $(p \times n)$ матрич, пусть $L \subset \mathbb{R}_{n}^{1}-$ порождающее $\mathscr{L}$ линейное подпространство в пространстве $n$-строк. Тогда для всякого $\lambda \in \mathbb{R}_{1}^{p}$

$$
\lambda^{T} \mathscr{L}=L
$$

Д о к а 3 а т е л ь с т в о л е м м ы. Пусть $r=\operatorname{dim} L, r \leqslant n$. Выберем в $L$ базис $e_{1}, \ldots, e_{r}$. Как мы знаем, подпространство $\mathscr{L} \in \mathbb{R}_{n}^{p}$ представимо как

$$
\mathscr{L}=\left\{y \mid y=\sum_{k=1}^{r} \alpha_{k} e_{k}, \alpha_{1}, \ldots, \alpha_{r} \in \mathbb{R}_{1}^{p}\right\} .
$$

Пусть $y \in \mathscr{L}$ и потому при некоторых $\alpha_{1}, \ldots, \alpha_{r} \in \mathbb{R}_{1}^{p}$

$$
y=\sum_{k=1}^{r} \alpha_{k} e_{k}
$$

Отсюда следует, что при всяком $\lambda \in \mathbb{R}_{1}^{p}$

$$
\lambda^{T} y=\sum_{k=1}^{r}\left(\lambda^{T} \alpha_{k}\right) e_{k} \in L,
$$

так как $\lambda^{T} \alpha_{1}, \ldots, \lambda^{T} \alpha_{r}$ - это числовые коэффициенты. Лемма 1 доказана. 
Теорема 4. Пусть $\mathscr{L} \subset \mathbb{R}_{n}^{p}-$ подмодуль в пространстве $(p \times n)$ матрии, пусть $L \subset \mathbb{R}_{n}^{1}-$ порождающее $\mathscr{L}$ линейное подпространство в пространстве $n$-строк. Пусть $\Pi-(n \times n)$-матрича оператора проектирования на L в пространстве $\mathbb{R}_{n}^{1}$ : для любого $x \in \mathbb{R}_{n}^{1}$

$$
\underset{L}{\operatorname{proj}} x=x \Pi \text {. }
$$

Тогда для любого $X \in \mathbb{R}_{n}^{p}$

$$
\underset{\mathscr{L}}{\operatorname{proj}} x=x \Pi .
$$

Д о к а з а т е л ь с т в о. Надо показать, что

$$
\langle x-y, x-y\rangle \succcurlyeq\langle x-x \Pi, x-x \Pi\rangle
$$

для любого $y \in \mathscr{L}$, причем равенство достигается только при $y=X \Pi$. Соотношение (1.14) между двумя симметрическими $(p \times p)$-матрицами означает, что для любого $\lambda \in \mathbb{R}_{1}^{p}$

$$
\lambda^{T}\langle x-y, x-y\rangle \lambda \geqslant \lambda^{T}\langle x-x \Pi, x-x \Pi\rangle \lambda,
$$

или

$$
\left|\lambda^{T}(x-y)\right|^{2} \geqslant\left|\lambda^{T}(x-x \Pi)\right|^{2},
$$

или

$$
\left|\lambda^{T} X-\lambda^{T} y\right|^{2} \geqslant\left|\lambda^{T} X-\left(\lambda^{T} x\right) \Pi\right|^{2} .
$$

Как было отмечено выше, $n$-строка $y=\lambda^{T} y$ принадлежит $L$, причем $\lambda^{T} X \Pi=x \Pi-$ это проекция $\lambda^{T} X$ на $L$. $\mathrm{B}$ силу свойств евклидовых проекций $|x-y|^{2} \geqslant|x-x \Pi|^{2}$ для всякого $y \in L$, причем равенство достигается только при $y=x \Pi$. Таким образом, $X \Pi$ действительно является ближайшей к $X$ точкой из $\mathscr{L}$. Теорема 4 доказана.

Для доказательства теоремы 3 остается показать, что $X-X \Pi$ ортогонально $\mathscr{L}$. Явный вид $\operatorname{proj}_{\mathscr{L}} X$ показывает, что операция проектирования на подмодуль - действительно линейная операция. Пусть $e_{1}, \ldots, e_{r}$ - ортогональный базис $L, e_{r+1}, \ldots, e_{n}$ - ортогональный базис $L^{\perp}$. Таким образом, $e_{1}, \ldots, e_{n}$ - ортогональный базис $\mathbb{R}_{n}^{1}$. Тогда утверждение - что $(X-X \Pi) \perp \mathscr{L}$ - доказываем прямой выкладкой, а именно: если в выбранном ортогональном порождающем базисе $\mathcal{X}=\sum_{i=1}^{n} \alpha_{i} e_{i}$, то $\mathcal{X}-X \Pi=\sum_{i=r+1}^{n} \alpha_{i} e_{i}$. В то же время общий вид $y \in \mathscr{L}$ есть $y=\sum_{i=1}^{r} \beta_{i} e_{i}$ при некотором выборе $\beta_{1}, \ldots, \beta_{r} \in \mathbb{R}_{1}^{p}$. Отсюда получаем:

$$
\begin{aligned}
(X-x \Pi) y^{T} & =\left\langle\sum_{i=r+1}^{n} \alpha_{i} e_{i}, \sum_{i=1}^{r} \beta_{i} e_{i}\right\rangle=\sum_{i=r+1}^{n} \sum_{j=1}^{r}\left\langle\alpha_{i} e_{i}, \beta_{j} e_{j}\right\rangle \\
& =\sum_{i=r+1}^{n} \sum_{j=1}^{r} \alpha_{i} e_{i}\left(\beta_{j} e_{j}\right)^{T}=\sum_{i=r+1}^{n} \sum_{j=1}^{r} \alpha_{i} e_{i} e_{j}^{T} \beta_{j}^{T}=0,
\end{aligned}
$$

так как $e_{i} e_{j}^{T}=0$ при $i \neq j$. 
1.6. Матричный метод наименьших квадратов. Вычисления проекций на подмодуль $\mathscr{L} \subset \mathbb{R}_{n}^{p}$ облегчаются, если известен вид проекций на порождающее $\mathscr{L}$ линейное подпространство $L$. Согласно лемме 1 из п. 1.5, для любого $\lambda \in \mathbb{R}_{1}^{p}$

$$
\lambda^{T} \operatorname{proj}_{\mathscr{L}} X=\operatorname{proj}_{L}\left(\lambda^{T} x\right) .
$$

Предположим, что для правой части (1.15) мы располагаем явной формулой $y=\operatorname{proj}_{L} x$. Тогда, в силу линейности операций, это даст нам для $\operatorname{proj}_{\mathscr{L}}\left(\lambda^{T} X\right)$ явную формулу вида $\lambda^{T} y$. Отсюда

$$
\lambda^{T} \underset{\mathscr{L}}{\operatorname{proj}} x=\lambda^{T} y .
$$

Так мы получаем явное выражение для $\operatorname{proj}_{\mathscr{L}} \mathcal{X}$. Можно сказать, что это - вычисление $\operatorname{proj}_{\mathscr{L}} X$ методом Роя [5].

П р и м е р (вычисление среднего арифметического). Пусть $X_{1}, \ldots, X_{n} \in \mathbb{R}_{1}^{p}$ - совокупность $p$-столбцов. Рассмотрим таблицу $\mathbf{T}=\left\{X_{i} \mid i=1, \ldots, n\right\}$ и представим ее в матричной форме

$$
\chi=\left\|X_{1}, \ldots, X_{n}\right\| .
$$

Наша задача - найти таблицу у с одинаковыми столбцами, т.е. таблицу вида

$$
y=\|Y, \ldots, Y\|, \quad Y \in \mathbb{R}_{1}^{p},
$$

ближайшую к (1.16). Таблицы вида (1.17) образуют одномерный подмодуль. Обозначим его $\mathscr{L} \subset \mathbb{R}_{n}^{p}$. Надо, следовательно, найти $\operatorname{proj}_{\mathscr{L}} X$. Подпространство $\mathscr{L}$ порождает одномерное линейное подпространство $L \subset \mathbb{R}_{n}^{1}$, натянутое на $n$-строку $e=(1, \ldots, 1)$.

Пусть $x$ - произвольная $n$-строка, $x=\left(x_{1}, \ldots, x_{n}\right)$. Вид проекции $x$ на $L$ хорошо известен:

$$
\underset{L}{\operatorname{proj}} x=(\bar{x}, \ldots, \bar{x}) .
$$

Применяя метод Роя, от матрицы $X(1.16)$ перейдем к $n$-строке $x=\lambda^{T} X$, при этом $x_{i}=\lambda^{T} X_{i}, i=1, \ldots, n$. Получим, что

$$
\underset{L}{\operatorname{proj}} x=\left(\lambda^{T} \bar{X}, \ldots, \lambda^{T} \bar{X}\right) \text {. }
$$

Следовательно,

$$
\underset{\mathscr{L}}{\operatorname{proj}} x=(\bar{X}, \ldots, \bar{X}) .
$$

Разумеется, это не единственный и не всегда наиболее экономный метод. В этом примере, как и во многих других случаях, можно применить матричный метод наименьших квадратов непосредственно и найти

$$
\widehat{Y}=\arg \min _{Y \in \mathbb{R}_{1}^{p}} \sum_{i=1}^{n}\left(X_{i}-Y\right)\left(X_{i}-Y\right)^{T} .
$$


Р е ш е н и е. Преобразуем целевую функцию: для произвольного $Y \in \mathbb{R}_{1}^{p}$

$$
\begin{aligned}
& \sum_{i=1}^{n}\left(X_{i}-Y\right)\left(X_{i}-Y\right)^{T}=\sum_{i=1}^{n}\left[\left(X_{i}-\bar{X}\right)+(\bar{X}-Y)\right]\left[\left(X_{i}-\bar{X}\right)+(\bar{X}-Y)\right]^{T} \\
& \quad=\sum_{i=1}^{n}\left(X_{i}-\bar{X}\right)\left(X_{i}-\bar{X}\right)^{T}+n \sum_{i=1}^{n}(\bar{X}-Y)(\bar{X}-Y)^{T}=(\mathrm{I})+(\mathrm{II}), \quad(1.19)
\end{aligned}
$$

так как «попарные произведения» обращаются в нуль:

$$
\sum_{i=1}^{n}\left(X_{i}-\bar{X}\right)(\bar{X}-Y)^{T}=0, \quad \sum_{i=1}^{n}(\bar{X}-Y)\left(X_{i}-\bar{X}\right)^{T}=0 .
$$

Теперь целевая функция представляет собой сумму двух неотрицательно определенных матриц, причем первая из них не зависит от изменений $Y$. Минимальный результат мы получим, положив $Y=\bar{X}$ : это обращает в нуль неотрицательно определенную матрицу (II).

Этот ответ $\widehat{Y}=\bar{X}$ (среднее арифметическое), конечно, хорошо известен. Его получают, применяя не матричный, но обычный метод наименьших квадратов, т.е. решая экстремальную задачу

$$
\widehat{Y}=\arg \min _{Y \in \mathbb{R}_{1}^{p}} \sum_{i=1}^{n}\left(X_{i}-Y\right)^{T}\left(X_{i}-Y\right) .
$$

Матричный и скалярный методы наименьших квадратов. Так же соотносятся результаты матричного и традиционного методов наименьших квадратов в случае проектирования на иные подмодули $\mathscr{L} \subset \mathbb{R}_{n}^{p}$. Причина проста: если таблица $y$ есть решение матричной задачи

$$
\sum_{i=1}^{n}\left(X_{i}-Z_{i}\right)\left(X_{i}-Z_{i}\right)^{T}=(X-z)(x-z)^{T} \rightarrow \min _{Z \in \mathscr{L}}
$$

то $y$ дает решение и скалярной задачи

$$
\operatorname{tr}\left\{\sum_{i=1}^{n}\left(X_{i}-Z_{i}\right)\left(X_{i}-Z_{i}\right)^{T}\right\}=\sum_{i=1}^{n}\left(X_{i}-Z_{i}\right)^{T}\left(X_{i}-Z_{i}\right) \rightarrow \min _{Z \in \mathscr{L}} .
$$

Поэтому, в частности, при вычислении проекций на подмодули можно обходиться традиционным скалярным методом наименьших квадратов. Оба метода наименьших квадратов - и матричный, и скалярный - в линейных моделях дают одинаковые ответы (одинаковые оценки параметров).

Необходимость матричных скалярных произведений и матричные понятия ортогональности, проектирования, подмодулей и прочего обнаруживается при статистической проверке линейных гипотез. Об этом в следующем разделе. 


\section{2. Многомерные линейные модели.}

2.1. Таблицы со случайными элементами. Рассмотрим таблицу, элементы которой представляют собой $p$-мерные случайные величины, записанные в виде $p$-столбцов:

$$
\mathbf{T}=\left\{X_{i} \mid i=1, \ldots, n\right\} .
$$

Напомним, что такую таблицу при алгебраических операциях мы воспринимаем как строку, элементами которой являются p-столбцы.

Для таблиц вида (2.1) со случайными элементами определим математические ожидания и ковариации. Математическим ожиданием $\mathbf{T}$ назовем таблицу

$$
\mathbf{E} \mathbf{T}_{X}=\left\{\mathbf{E} X_{i}, i=1, \ldots, n\right\} .
$$

Матрицу ковариаций для (2.1) определим, следуя образцу, который дает матрица ковариаций случайного вектора. Пусть $t=\left(x_{1}, \ldots, x_{n}\right)-n$ строка, составленная из случайных величин $x_{1}, \ldots, x_{n}$. Как известно, матрицей ковариаций случайного вектора $t$ называют $(n \times n)$-матрицу с элементами $\sigma_{i j}=\operatorname{Cov}\left(x_{i}, x_{j}\right)$, где $i, j=1, \ldots, n$. Алгебраически, с помощью матричных операций, матрицу ковариаций вектора $t$ можно определить как

$$
\operatorname{Var} t=\mathbf{E}(t-\mathbf{E} t)^{T}(t-\mathbf{E} t) .
$$

Следуя (2.3), таблицей ковариаций случайной таблицы (2.1) мы назовем таблицу

$$
\operatorname{Var} \mathbf{T}:=\mathbf{E}(\mathbf{T}-\mathbf{E} \mathbf{T})^{T}(\mathbf{T}-\mathbf{E} \mathbf{T})=\left\{\operatorname{Cov}\left(X_{i}, X_{j}\right) \mid i, j=1, \ldots, n\right\} .
$$

Здесь $\operatorname{Cov}\left(X_{i}, X_{j}\right)$ - матрица ковариаций случайных векторов-столбцов $X_{i}$ и $X_{j}$ :

$$
\operatorname{Cov}\left(X_{i}, X_{j}\right)=\mathbf{E}\left(X_{i}-\mathbf{E} X_{i}\right)\left(X_{j}-\mathbf{E} X_{j}\right)^{T} .
$$

Отметим, что $\operatorname{var} \mathbf{T}(2.4)$ мы воспринимаем как квадратную таблицу размера $n \times n$, элементы которой - это $(p \times p)$-матрицы $(2.5)$. Именно такого взгляда на (2.4) мы будем держаться, когда будем обсуждать преобразования таблиц ковариаций при линейных преобразованиях случайных таблиц.

Рассмотрим таблицу $\mathbf{R}$, полученную линейным преобразованием таблицы T (2.1):

$$
\mathbf{R}=\mathbf{T} Q,
$$

где $Q$ - некоторая $(n \times n)$-матрица.

Очевидно, что для математических ожиданий таблиц действует обычное правило линейности: $\mathbf{E} \mathbf{R}=(\mathbf{E} \mathbf{T}) Q$. Для $\operatorname{var} \mathbf{R}$, выполняя перемножения таблиц (и матриц) по правилу «строка на столбец», получим

$$
\operatorname{Var} \mathbf{R}=\mathbf{E}\left[((\mathbf{T}-\mathbf{E} \mathbf{T}) Q)^{T}((\mathbf{T}-\mathbf{E} \mathbf{T}) Q)\right]=Q^{T}(\operatorname{Var} \mathbf{T}) Q .
$$


Для математической статистики особый интерес представляют таблицы со статистически независимыми случайными элементами, когда матрицы ковариаций этих элементов одинаковы. Пусть Т (2.1) - такая таблица, так что

$$
\operatorname{Cov}\left(X_{i}, X_{j}\right)=\delta_{i j} \Sigma \quad \text { для } i, j=1, \ldots, n .
$$

Здесь $\Sigma-$ некоторая неотрицательно определенная $(p \times p)$-матрица, $\delta_{i j}$ - символ Кронекера. Рассмотрим ортогональное преобразование таблицы $\mathbf{T}$ :

$$
\mathbf{R}=\mathbf{T} C,
$$

где $C$ - некоторая ортогональная $(n \times n)$-матрица. Справедлива простая, но важная лемма.

Лемма 2. При ортогональньх преобразованиях вариачия таблиць сохраняется:

$$
\operatorname{Var} \mathbf{R}=\operatorname{Var} \mathbf{T}=\left\{\delta_{i j} \Sigma \mid i, j=1, \ldots, n\right\}
$$

Формула (2.10) показывает, что при ортогональных преобразованиях таблиц с некоррелированными элементами с одинаковыми ковариациями появляются случайные таблицы, элементы которых тоже попарно некоррелированы, ковариации которых одинаковы и совпадают с ковариацией элементов исходной таблицы.

Д о к а з а т е л ь с т в о. Доказательство теоремы - простая выкладка. Чтобы упростить формулы, предположим, что $\mathbf{E} \mathbf{T}=0$. Тогда, в силу $(2.7)$,

$$
\begin{aligned}
\mathbf{E}(\mathbf{T} C) & =\mathbf{E}\left[(\mathbf{T} C)^{T}(\mathbf{T} C)\right]=C^{T}(\operatorname{Var} \mathbf{T}) C \\
& =C^{T}\left\{\delta_{i j} \Sigma \mid i, j=1, \ldots, n\right\} C=\left\{\delta_{i j} \Sigma \mid i, j=1, \ldots, n\right\} .
\end{aligned}
$$

Лемма 2 доказана.

$\mathrm{K}$ сожалению, у нас в запасе нет алгебраической операции, которая при умножении единичной $(n \times n)$-матрицы, воспринимаемой как таблица, на матрицу $\Sigma$, давала бы сразу таблицу (2.10). С использованием такой операции выкладка была бы особенно выразительна: была бы полная аналогия в формулировке и доказательстве с аналогичным утверждением о случайных векторах.

Ранее, при обсуждении порождающих базисов и координат (п. 1.3.), мы установили, что переход от координат таблицы $\mathbf{T}$ в одном ортогональном базисе к координатам этой таблицы в другом базисе осуществляется умножением на ортогональную матрицу. Из этого замечания и только что установленной леммы 2 вытекает следующее утверждение. 
Лемма 3. Если координатьи некоторой таблииь в одном ортогональном базисе некоррелировань и имеют общую матричу ковариачий, то это свойство координаты данной таблицы имеют в любом ортогональном базисе.

Этот результат хорошо известен в других редакциях (см., например, [3, теорема 3.3]). Лемма 3 будет важна при изучении линейных статистических моделей.

Наконец, отметим, что при введении и обсуждении таблиц ковариаций для случайных таблиц нам необходимо оперировать с самими таблицами (1.1), а не - как часто бывало - с представляющими их матрицами (1.7).

\section{2. Линейные модели и линейные гипотезы.}

О п р е д е л е н и е 3 . Говорят, что таблица $\mathbf{T}(2.1)$ со случайными элементами следует линейной модели, если а) для некоторого заданного подмодуля $\mathscr{L}$

$$
\mathbf{E} \mathbf{T} \in \mathscr{L}
$$

b) элементы $X_{1}, \ldots, X_{n}$ таблицы $\mathbf{T}$ независимы и одинаково распределены.

Если это общее для всех $X_{i}, i=1, \ldots, n$, распределение гауссовское, то о таблице $\mathbf{T}$ говорят, что она следует линейной гауссовской модели. Далее мы будем изучать линейные гауссовские модели.

Общую для всех $p$-столбцов матрицу ковариаций обозначим $\Sigma$. Таблица Е Т и матрица $\Sigma$ являются параметрами модели. Они обычно неизвестны; матрица $\Sigma$ предполагается невырожденной.

Для случайных таблиц, следующих гауссовской модели, часто обсуждают особый тип гипотез, называемых линейньми гипотезами. В рамках линейной модели (2.11) линейная гипотеза имеет вид

$$
\mathbf{E} \mathbf{T} \in \mathscr{L}_{1}
$$

где $\mathscr{L}_{1}$ - некоторый заданный подмодуль, причем $\mathscr{L}_{1} \subset \mathscr{L}$.

Покажем, что обсуждаемые в многомерном статистическом анализе линейные модели и линейные гипотезы действительно имеют структуру (2.11) и (2.12). Основные линейные модели - факторные и регрессионные. Как примеры мы рассмотрим однофакторную и регрессионную модели многомерного статистического анализа.

Однофакторная модель - простейшая среди факторных моделей. Это задача о нескольких (скажем, $m$ ) нормальных выборках, в которой матрицы ковариаций отдельных наблюдений одинаковы. Таблица данных в этом случае имеет двойную нумерацию:

$$
\mathbf{T}=\left\{X_{i j} \mid j=1, \ldots, m, i=1, \ldots, n_{j}\right\} .
$$


Здесь $m$ - число различных уровней некоего фактора, оказывающего влияние на ожидаемые значения откликов, $n_{j}$ - количество независимых повторных наблюдений на уровне фактора с номером $j=1, \ldots, m$. Наконец, многомерные случайные переменные $X_{i j}$ - это независимые (при разных значениях индекса) значения $p$-мерного отклика, $X_{i j} \in \mathbb{R}_{1}^{p}$. Положим $N=n_{1}+\cdots+n_{m}$. Основное предположение модели: $X_{i j} \sim$ $N_{p}\left(a_{j}, \Sigma\right)$.

Наблюдения, составляющие таблицу (2.13), расположим линейно и далее будем представлять совокупность данных $(2.13)$ в виде $(p \times N)$ матрицы

$$
X=\left\|X_{11}, \ldots, X_{1 n_{1}}, X_{21}, \ldots, X_{2 n_{2}}, X_{m 1}, \ldots X_{m n_{m}}\right\| .
$$

При этом $\mathbf{E} X=\|\underbrace{a_{1}, \ldots, a_{1}}_{n_{1} \text { раз }}, \underbrace{a_{2}, \ldots, a_{2}}_{n_{2} \text { раз }}, \ldots, \underbrace{a_{m}, \ldots, a_{m}}_{n_{m} \text { раз }}\|$. Введем $N$-строки

$$
e_{1}=(\underbrace{1, \ldots, 1}_{n_{1} \text { раз }}, 0, \ldots, 0), \ldots, e_{m}=(\underbrace{0, \ldots, 0}_{n_{1} \text { раз }}, \underbrace{0, \ldots, 0}_{n_{2} \text { раз }}, \ldots, \underbrace{1, \ldots, 1}_{n_{m} \text { раз }}) \text {. }
$$

Очевидно, что $\mathbf{E} X=\sum_{i=1}^{m} a_{i} e_{i}$. Тем самым, $\mathbf{E} X$ принадлежит $m$-мерному подмодулю пространства $\mathbb{R}_{N}^{p}$, которое порождено $N$-строками (2.15).

Гипотеза $H_{0}: a_{1}=\cdots=a_{m}$, с проверки которой обычно начинают статистический анализ $m$ выборок, очевидно, является линейной гипотезой в смысле (2.12): она означает, что $\mathbf{E} X \in \mathscr{L}_{1}$, где $\mathscr{L}_{1}$ - одномерное линейное подпространство, порожденное единственной $N$-строкой $e=e_{1}+\cdots+e_{m}$.

Многомерная множественная регрессия в своей матричной форме имеет вид

$$
y=\mathcal{A} X+\mathcal{E},
$$

где $y=\left\|Y_{1}, \ldots, Y_{n}\right\|$ - наблюдаемая $(p \times n)$-матрица $p$-мерных откликов, $\mathcal{X}$ - заданная $(m \times n)$-матрица плана, $\mathcal{A}-(p \times m)$-матрица неизвестных коэффициентов регрессии, $\mathcal{E}=\left\|E_{1}, \ldots, E_{n}\right\|-(p \times n)$-матрица, составленная из $p$-столбцов $E_{1}, \ldots, E_{n}$ независимых случайных ошибок. В гауссовской модели $E_{i} \sim N_{p}(0, \Sigma)$, где $(p \times p)$-матрица $\Sigma$ предполагается невырожденной. Обычно $\Sigma$ считается неизвестной.

Пусть $A_{1}, \ldots, A_{m}$ суть $p$-столбцы, составляющие матрицу $\mathcal{A} ;$ пусть $x_{1}, \ldots, x_{m}-n$-строки, составляющие матрицу плана $\mathcal{X}$. Тогда

$$
\mathcal{A X}=\sum_{i=1}^{m} A_{i} x_{i}
$$

Полученное выражение (2.17) показывает, что $\mathbf{E} y=\mathcal{A} X$ принадлежит $m$-мерному линейному подпространству пространства $\mathbb{R}_{n}^{p}$, порожденному линейной системой $n$-строк $x_{1}, \ldots, x_{m}$. 
2.3. Достаточные статистики, наилучшие несмещенные оценки. Рассмотрим линейную гауссовскую модель (2.11). Представим ее в матричной форме

$$
x=\mathcal{M}+\mathcal{E},
$$

где $\mathcal{M}=\mathbf{E} \mathcal{X}$ - неизвестная $(p \times n)$-матрица, $\mathcal{M}=\left\|M_{1}, \ldots, M_{n}\right\| \in \mathscr{L}$ (здесь $\mathscr{L}$ - некоторый заданный подмодуль $\mathbb{R}_{n}^{p}$ ), и $\mathcal{E}=\left\|E_{1}, \ldots, E_{n}\right\|-$ $(p \times n)$-матрица, $p$-столбцы $E_{1}, \ldots, E_{n}$ которой суть независимые $p$ мерные случайные величины с распределением $N_{p}(0, \Sigma)$.

Параметром (неизвестным) этой гауссовской модели служит пара $(\mathcal{M}, \Sigma)$. Найдем для этого параметра достаточную статистику (с помощью теоремы факторизации).

Правдоподобие пары $\mathcal{M}, \Sigma$ по наблюдению $\mathcal{X}$ равно

$$
\begin{aligned}
\prod_{i=1}^{n} & \left(\frac{1}{\sqrt{2 \pi}}\right)^{p} \frac{1}{\sqrt{\operatorname{det} \Sigma}} \exp \left\{-\frac{1}{2}\left(X_{i}-M_{i}\right)^{T} \Sigma^{-1}\left(X_{i}-M_{i}\right)\right\} \\
= & \left(\frac{1}{\sqrt{2 \pi}}\right)^{n p}\left(\frac{1}{\sqrt{\operatorname{det} \Sigma}}\right)^{n} \\
& \times \exp \left\{-\frac{1}{2} \operatorname{tr} \Sigma^{-1}\left[\sum_{i=1}^{n}\left(X_{i}-M_{i}\right)\left(X_{i}-M_{i}\right)^{T}\right]\right\} .
\end{aligned}
$$

Сумма в квадратных скобках - это $\langle x-\mathcal{M}, x-\mathcal{M}\rangle$. Представим $\mathcal{X}-\mathcal{M}$ в виде $X-\mathcal{M}=\left(X-\operatorname{proj}_{\mathscr{L}} X\right)+\left(\operatorname{proj}_{\mathscr{L}} X-\mathcal{M}\right)=(\mathrm{I})+(\mathrm{II})$ и заметим, что $(\mathrm{I})=\operatorname{proj}_{\mathscr{L} \perp} \mathcal{X} \in \mathscr{L}^{\perp},(\mathrm{II}) \in \mathscr{L}$. Поэтому (теорема Пифагора)

$$
\langle x-\mathcal{M}, x-\mathcal{M}\rangle=\left\langle\operatorname{proj}_{\mathscr{L} \perp} x, \operatorname{proj}_{\mathscr{L} \perp} x\right\rangle+\left\langle\operatorname{proj}_{\mathscr{L}} x-\mathcal{M}, \operatorname{proj}_{\mathscr{L}} x-\mathcal{M}\right\rangle .
$$

Заключаем отсюда, что правдоподобие (2.19) выражается через статистики $\operatorname{proj}_{\mathscr{L}} X$ и $\left\langle\operatorname{proj}_{\mathscr{L} \perp} X, \operatorname{proj}_{\mathscr{L} \perp} X\right\rangle$, которые вместе достаточны для $\mathcal{M}, \Sigma$.

Статистика $\operatorname{proj}_{\mathscr{L}} \mathcal{X}$, очевидно, является несмещенной оценкой $\mathcal{M}$. Как функция достаточной статистики она является наилучшей несмещенной оценкой $\mathcal{M}$. То, что наилучшей несмещенной оценкой $\Sigma$ служит статистика

$$
\frac{1}{\operatorname{dim} \mathscr{L}^{\perp}}\left\langle\operatorname{proj}_{\mathscr{L} \perp} x, \operatorname{proj}_{\mathscr{L} \perp} x\right\rangle
$$

мы установим после доказательства следующей далее теоремы 5.

\section{4. Теорема об ортогональном разложении.}

Теорема 5. Пусть $x=\left\|X_{1}, \ldots, X_{n}\right\|-$ гауссовская $(p \times n)$ матрича с независимьми р-столбиами $X_{1}, \ldots, X_{n} \in \mathbb{R}_{1}^{p}$, причем $\operatorname{Var} X_{i}=\Sigma$ для всех $i=1, \ldots, n$. Пусть $\mathscr{L}_{1}, \mathscr{L}_{2}, \ldots-$ попарно ортогональные подмодули $\mathbb{R}_{n}^{p}$, прямая сумма которых составляет $\mathbb{R}_{n}^{p}$ :

$$
\mathbb{R}_{n}^{p}=\mathscr{L}_{1} \oplus \mathscr{L}_{2} \oplus \cdots .
$$


Рассмотрим разложение $(p \times n)$-матричь $X$ на попарно ортогональные проекиии $X$ на подмодули $\mathscr{L}_{1}, \mathscr{L}_{2}, \ldots: X=\operatorname{proj}_{\mathscr{L}_{1}} X+\operatorname{proj}_{\mathscr{L}_{2}} X+\cdots$.

Тогда:

а) случайнье $(p \times n)$-матричьи $\operatorname{proj}_{\mathscr{L}_{1}} X, \operatorname{proj}_{\mathscr{L}_{2}} x, \ldots$ независимьи, $р а с-$ пределеньи нормально и $\mathbf{E} \operatorname{proj}_{\mathscr{L}_{i}} X=\operatorname{proj}_{\mathscr{L}_{i}} \mathbf{E} X$;

b) $\left\langle\operatorname{proj}_{\mathscr{L}_{i}} x, \operatorname{proj}_{\mathscr{L}_{i}} x\right\rangle=W_{p}\left(\operatorname{dim} L_{i}, \Sigma, \Delta_{i}\right)$, где $W_{p}(\nu, \Sigma, \Delta)$ обозначает случайную матричу (размера $p \times p$ ), распределенную по Уишарту,

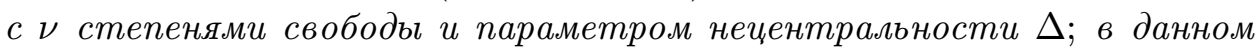
случае $\Delta_{i}=\left\langle\operatorname{proj}_{\mathscr{L}_{i}} \mathbf{E} X, \operatorname{proj}_{\mathscr{L}_{i}} \mathbf{E} X\right\rangle$.

Д о к а з а т е л ь с т в о. Каждому подмодулю $\mathscr{L} \subset \mathbb{R}_{n}^{p}$ в пространстве $\mathbb{R}_{n}^{1}$ взаимно однозначно соответствует некоторое порождающее его линейное подпространство $L \subset \mathbb{R}_{n}^{1}$, причем $\operatorname{dim} \mathscr{L}=\operatorname{dim} L$. Пусть подмодулям $\mathscr{L}_{1}, \mathscr{L}_{2}, \ldots \subset \mathbb{R}_{n}^{p}$ соответствуют подпространства $L_{1}, L_{2}, \ldots \subset \mathbb{R}_{n}^{1}$. При этом подпространства $L_{1}, L_{2}, \ldots \subset \mathbb{R}_{n}^{1}$ попарно ортогональны и их прямая сумма образует все пространство $\mathbb{R}_{n}^{1}$. Обозначим размерности подмодулей $\mathscr{L}_{1}, \mathscr{L}_{2}, \ldots \subset \mathbb{R}_{n}^{p}$ (и подпространств $\left.L_{1}, L_{2}, \ldots \subset \mathbb{R}_{n}^{1}\right)$ через $m_{1}, m_{2}, \ldots$.

Выберем в каждом подпространстве $L_{1}, L_{2}, \ldots$ ортогональный базис. Пусть для $\mathscr{L}_{1}$ это $n$-строки $f_{1}, \ldots, f_{m_{1}}$; для $\mathscr{L}_{2}-$ это $n$-строки $f_{m_{1}+1}, \ldots, f_{m_{1}+m_{2}}$ и т.д. С помощью этих $n$-строк каждое из подпространств $\mathscr{L}_{1}, \mathscr{L}_{2}, \ldots$ можно представить как прямую сумму одномерных подпространств из $\mathbb{R}_{n}^{p}$. Например, $\mathscr{L}_{1}=\mathscr{F}_{1} \oplus \cdots \oplus \mathscr{F}_{m_{1}}$, где

$$
\mathscr{F}_{j}=\left\{y \mid y=\alpha f_{j}, \alpha \text { пробегает } \mathbb{R}_{1}^{p}\right\}, \quad j=1, \ldots, m_{1} .
$$

Совокупность всех $n$-строк $f_{1}, \ldots, f_{n}$ образует ортогональный базис $\mathbb{R}_{n}^{1}$ и, одновременно, порождающий базис пространства $\mathbb{R}_{n}^{p}$. Поэтому всякую $(p \times n)$-матрицу $\chi \in \mathbb{R}_{n}^{p}$ можно представить в виде

$$
X=\sum_{i=1}^{n} Y_{i} f_{i},
$$

где $Y_{1}, \ldots, Y_{n}$ - некоторые $p$-столбцы, т.е. $Y_{1}, \ldots, Y_{n} \in \mathbb{R}_{1}^{p}$. При этом

$$
\operatorname{proj}_{\mathscr{L}_{1}} X=\sum_{i=1}^{m_{1}} Y_{i} f_{i}, \quad \operatorname{proj}_{\mathscr{L}_{2}} X=\sum_{i=m_{1}+1}^{m_{2}} Y_{i} f_{i} \quad \text { и т.д. }
$$

Здесь $p$-столбцы $Y_{1}, \ldots, Y_{n}$ - это координаты $(p \times n)$-матрицы $X$ относительно порождающего базиса $f_{1}, \ldots, f_{n}$. При этом $p$-столбцы $X_{1}, \ldots, X_{n}$ - это координаты той же $(p \times n)$-матрицы $X$ относительно ортогонального канонического базиса $\mathbb{R}_{n}^{1}: e_{1}=(1,0, \ldots), e_{2}=(0,1,0, \ldots)$ и т.д. Как было отмечено ранее (п. 1.3), переход от одних координат к другим осуществляется умножением $(p \times n)$-матрицы $X$ справа на $(n \times n)$-матрицу перехода, в данном случае - на некоторую ортогональную $(n \times n)$-матрицу $C:\left\|Y_{1}, \ldots, Y_{n}\right\|=\left\|X_{1}, \ldots, X_{n}\right\| C$, или $y=X C$. 
Из этого следует, что $p$-столбцы $Y_{1}, \ldots, Y_{n}$ в совокупности распределены нормально. Затем, согласно лемме 2 из п. 2.1,

$$
\operatorname{Var} y=\operatorname{Var} X=\left\{\delta_{i j} \Sigma \mid i, j=1, \ldots, n\right\} .
$$

Это значит, что $p$-столбцы $Y_{1}, \ldots, Y_{n}$ статистически независимы и имеют $(p \times p)$-матрицу ковариаций $\Sigma$ такую же, как $p$-столбцы $X_{1}, \ldots, X_{n}$.

Рассмотрим случайные $(p \times p)$-матрицы $\left\langle\operatorname{proj}_{\mathscr{L}_{1}} x, \operatorname{proj}_{\mathscr{L}_{1}} x\right\rangle,\left\langle\operatorname{proj}_{\mathscr{L}_{2}} x\right.$, $\left.\operatorname{proj}_{\mathscr{L}_{2}} X\right\rangle, \ldots$. Например,

$$
\left\langle\operatorname{proj}_{\mathscr{L}_{1}} x, \operatorname{proj}_{\mathscr{L}_{1}} x\right\rangle=\sum_{i=1}^{m_{1}} Y_{i} Y_{i}^{T}
$$

Распределения таких случайных матриц называют распределениями Уишарта. Если при этом $\mathbf{E} Y_{1}=\cdots=\mathbf{E} Y_{m_{1}}=0$, мы получаем так называемое центральное распределение Уишарта $W_{p}\left(m_{1}, \Sigma\right)$. Заметим, что если использовать обозначение $W_{p}(m, \Sigma)$ не только для закона распределения, но и для случайной матрицы с таким распределением, то можно сказать, что $W_{p}(m, \Sigma)=\Sigma^{1 / 2} W_{p}(m, I) \Sigma^{1 / 2}$, если обозначить $\Sigma^{1 / 2}$ симметрическую матрицу, единственное симметричное решение матричного уравнения $Z^{2}=\Sigma$.

Говорят, что случайная $(p \times p)$-матрица $W$ имеет нецентральное распределение Уишарта, если

$$
W=\sum_{i=1}^{m}\left(\xi_{i}+a_{i}\right)\left(\xi_{i}+a_{i}\right)^{T},
$$

где $p$-столбцы $\xi_{1}, \ldots, \xi_{m}$ независимы и имеют распределение $N_{p}(0, \Sigma)$, $a_{1}, \ldots, a_{m}$ - некоторые неслучайные $p$-столбцы, вообще говоря, отличные от нуля. Распределение $W$ каким-то образом зависит от $p$-столбцов $a_{1}, \ldots, a_{m}$. Покажем, что распределение $W$ зависит от упомянутых $p$ столбцов через так называемый параметр нецентральности - $(p \times p)$ матрицу

$$
\Delta=\sum_{i=1}^{m} a_{i} a_{i}^{T}
$$

Введем $(p \times m)$-матрицы $\xi=\left\|\xi_{1}, \ldots, \xi_{m}\right\|, \mathcal{A}=\left\|a_{1}, \ldots, a_{m}\right\|$. В этих обозначениях $W=\langle\xi+\mathcal{A}, \xi+\mathcal{A}\rangle$.

Пусть $C-$ произвольная ортогональная $(m \times m)$-матрица. Положим $\eta=\xi C$. При этом $\eta \stackrel{\mathrm{d}}{=} \xi$ и $W \stackrel{\mathrm{d}}{=}\langle\eta+A C, \eta+A C\rangle$. Мы видим, что нецентральное распределение Уишарта зависит от $\mathcal{A}=\left\|a_{1}, \ldots, a_{m}\right\|$ не непосредственно, но через максимальный инвариант $\mathcal{A}$ при ортогональных преобразованиях, т.е. через $\langle\mathcal{A}, \mathcal{A}\rangle=\sum_{i=1}^{m} a_{i} a_{i}^{T}$. 
Следовательно, в общем случае

$$
\left\langle\operatorname{proj}_{\mathscr{L}_{i}} x, \operatorname{proj}_{\mathscr{L}_{i}} x\right\rangle=W_{p}\left(m_{i}, \Sigma, \Delta_{i}\right),
$$

где $\Delta_{i}=\left\langle\operatorname{proj}_{\mathscr{L}_{i}} \mathbf{E} X, \operatorname{proj}_{\mathscr{L}_{i}} \mathbf{E} X\right\rangle$. Доказательство теоремы 5 закончено.

Вернемся к несмещенным оценкам параметров линейной модели. В линейной модели (2.18) $\operatorname{proj}_{\mathscr{L} \perp} \mathbf{E} X=0$. Поэтому статистика $(2.21)$ есть

$$
\frac{1}{\operatorname{dim} \mathscr{L}^{\perp}}\left\langle\operatorname{proj}_{\mathscr{L} \perp} X, \operatorname{proj}_{\mathscr{L} \perp} X\right\rangle=\frac{1}{n-m} \Sigma^{1 / 2} W_{p}(n-m, I) \Sigma^{1 / 2} .
$$

Очевидно, что ее математическое ожидание равно $\Sigma$.

2.5. Проверка линейных гипотез. Копируя одномерную линейную модель, линейной гипотезой в многомерной линейной модели (2.18) назовем гипотезу

$$
H: \mathbf{E} X \in \mathscr{L}_{1},
$$

где $\mathscr{L}_{1}-$ заданный подмодуль, причем $\mathscr{L}_{1} \subset \mathscr{L}$.

В этом пункте мы предложим статистики, которые могут служить основой для построения статистических критериев для проверки гипотезы $H(2.22)$, свободных (при $H$ ) от параметров $\mathcal{M}, \Sigma$.

Введем подмодуль $\mathscr{L}_{2}$, ортогонально дополняющий $\mathscr{L}_{1}$ до $\mathscr{L}$ :

$$
\mathscr{L}=\mathscr{L}_{1} \oplus \mathscr{L}_{2}
$$

Рассмотрим разложение пространства $\mathbb{R}_{n}^{p}$ на три попарно ортогональных подпространства: $\mathbb{R}_{n}^{p}=\mathscr{L}_{1} \oplus \mathscr{L}_{2} \oplus \mathscr{L}^{\perp}$. В силу теоремы 5 случайные матрицы

$$
S_{1}:=\left\langle\operatorname{proj}_{\mathscr{L} \perp} x, \operatorname{proj}_{\mathscr{L} \perp} x\right\rangle \quad \text { и } \quad S_{2}:=\left\langle\operatorname{proj}_{\mathscr{L}_{2}} x, \operatorname{proj}_{\mathscr{L}_{2}} x\right\rangle
$$

статистически независимы и распределены по Уишарту. При этом

$$
S_{1}=\left\langle\operatorname{proj}_{\mathscr{L} \perp} \mathcal{X}, \operatorname{proj}_{\mathscr{L} \perp} X\right\rangle=W_{p}(n-m, \Sigma) .
$$

Если гипотеза $H(2.22)$ верна, то

$$
S_{2}=\left\langle\operatorname{proj}_{\mathscr{L}_{2}} x, \operatorname{proj}_{\mathscr{L}_{2}} x\right\rangle=W_{p}\left(m_{2}, \Sigma\right)
$$

(Здесь и далее приняты обозначения $m=\operatorname{dim} \mathscr{L}, m_{1}=\operatorname{dim} \mathscr{L}_{1}, m_{2}=$ $\operatorname{dim} \mathscr{L}_{2}$.)

При нарушении гипотезы $H(2.22)$ распределение Уишарта статистики (2.25) становится нецентральным, приобретая параметр нецентральности $\Delta=\left\langle\operatorname{proj}_{\mathscr{L}_{2}} \mathbf{E} X, \operatorname{proj}_{\mathscr{L}_{2}} \mathbf{E} X\right\rangle$, который тем больше, чем «сильнее» нарушена гипотеза $H(2.22): \mathbf{E} X \in \mathscr{L}_{1}$.

В одномерном случае (когда $p=1$ ) статистики $(2.24),(2.25)$ превращаются в случайные величины, распределенные как $\sigma^{2} \chi^{2}(n-m)$ и 
$\sigma^{2} \chi^{2}\left(m_{2}\right)$. Их отношение (при гипотезе $H$ ) распределено свободно и потому может быть использовано как статистический критерий для проверки $H$. Это F-отношение Фишера.

В многомерном случае аналог $\mathrm{F}$-отношения - это отношение $(p \times p)$ матриц $S_{2}$ и $S_{1}$. При $n-m \geqslant p$ матрица $S_{1}(2.24)$ невырождена, и потому существует статистика $(p \times p$-матрица)

$$
\left\langle\operatorname{proj}_{\mathscr{L}_{2}} x, \operatorname{proj}_{\mathscr{L}_{2}} x\right\rangle\left\langle\operatorname{proj}_{\mathscr{L} \perp} x, \operatorname{proj}_{\mathscr{L} \perp} x\right\rangle^{-1} \text {. }
$$

В отличие от одномерного случая $(p=1)$ статистика $(2.26)$ не распределена свободно: по распределению она равна

$$
\Sigma^{1 / 2} W_{p}\left(m_{2}, I\right) W_{p}^{-1}(n-m, I) \Sigma^{-1 / 2}
$$

Однако собственные значения матрицы (2.26) при гипотезе $H(2.22)$ распределены свободно от $\mathcal{M}, \Sigma$. Эти собственные значения совпадают с корнями уравнения (относительно $\lambda$ )

$$
\operatorname{det}\left(W_{p}\left(m_{2}, I\right)-\lambda W_{p}(n-m, I)\right)=0 .
$$

Поэтому именно некоторые функции от корней уравнения (2.28) традиционно используют в качестве критических статистик для проверки линейных гипотез.

Здесь наше исследование выходит на традиционную дорогу многомерного статистического анализа и потому должно быть закончено.

Приношу благодарность Е. М. Сухановой, А. А. Саранцеву и П. С. Панову за обсуждения и помощь в работе.

\section{СПИСОК ЛИТЕРАТУРЫ}

1. Колмогоров А. Н. К обоснованию методов наименьших квадратов. - Успехи матем. наук, 1946, т. 1, в. 1, с. 57-70; перепеч. в: А. Н. Колмогоров. Теория вероятностей и математическая статистика (сб. статей). М.: Наука, 1986, с. 267-283.

2. Durbin J., Kendall M. G. The geometry of estimation. - Biometrika, 1951, v. 38, № $1 / 2$, p. $150-158$.

3. Андерсон Т. Введение в многомерный статистический анализ. М.: Физматгиз, $1963,500 \mathrm{c}$.

4. Шеффе Г. Дисперсионный анализ. М.: Физматгиз, 1963, 625 с.

5. Roy S. N. Some Aspects of Multivariate Analysis. New York: Wiley, 1957, 214 p.

6. Bilodeau M., Brenner D. Theory of Multivariate Statistics. New York: SpringerVerlag, 1999, $288 \mathrm{p}$.

7. Гантмахер Ф.Р. Теория матриц. М.: Физматлит, 2004, 560 с.

8. Horn R. A., Johnson C. R. Topics in Matrix Analysis. Cambridge: Cambridge Univ. Press, 1994, 615 p. 